\title{
Determinantes socioeconómicos del consumo de productos ecológicos en Quito
}

\section{Socioeconomic determinants of the consumption of ecological products in Quito}

\author{
Cristian Vasco ${ }^{1}$, Gabriela Palacios ${ }^{2}$, Susana Paspuel ${ }^{2}$ \\ ${ }^{1}$ Universidad Central del Ecuador. Facultad de Ciencias Agricolas. Jerónimo Leiton y Av. La Gasca s/n. Ciudadela Universitaria. 17052.1 Quito, Ecuador \\ ${ }^{2}$ Universidad de las Fuerzas Armadas-ESPE. Sangolqui, Ecuador
}

\begin{abstract}
Resumen
En este estudio se analizan los determinantes socioeconómicos del consumo de productos orgánicos y ecológicos en el Distrito Metropolitano de Quito. Para ello se realizaron encuestas entre los clientes de la feria agroecológica del parque "la Carolina" y se analizaron los datos por medio de una regresión lineal con el método de mínimos cuadrados ordinarios (OLS). Los resultados sugieren que las personas que poseen un mayor nivel de escolaridad así como más ingresos económicos, gastan más en el consumo de productos orgánicos y ecológicos. A la luz de estos resultados, las políticas orientadas a incrementar la oferta y la demanda de alimentos producidos ecológicamente deberían enfocarse en: 1) fomentar y promover la producción ecológica a fin de reducir los costos y el precio final al consumidor, y 2) difundir los beneficios y ventajas de consumir productos ecológicos entre los segmentos de la población con menos escolaridad e ingresos.
\end{abstract}

Palabras clave: alimentos ecológicos, mínimos cuadrados ordinarios, determinantes socioeconómicos, Quito.

\begin{abstract}
This work analyzes the socioeconomic determinants of the concumption of organic and ecological products in Quito. We conducted a survey among the buyers of an agroecological fair, which takes place in "La Carolina Park". For the analysis, we used a linear regression following an ordinary least squares approach (OLS). The results show that persons with higher incomes and higher endowments of education spend more on agroecological products. In the light of these results, policies should be oriented to increase the offer and the demand of ecological products should focus on 1) support and promote ecological production with the aim of reducing production costs and he final Price, and 2) disseminate the benefits and advantages of consuming ecological products among the segments of the population with low levels of income and schooling.
\end{abstract}

Keywords: ecological food, ordinary least squares, socioeconomic determinants, Quito.

\section{Introducción}

El impacto ambiental que ocasiona el excesivo uso de insecticidas, fungicidas, herbicidas y demás plaguicidas químicos utilizados en la agricultura convencional, ha traído consigo el deterioro del suelo, así como de los recursos acuáticos, donde a causado severos daños a la salud humana, entre los cuales se incluyen presencia de cáncer, aberraciones genéticas, desórdenes en el aprendizaje, problemas en el sistema inmunológico, alteraciones reproductivas, diabetes, entre otros (Badii \& Landeros, 2007). En este contexto, la agricultura ecológica se erige como una alternativa de producción limpia y consumo de productos sanos que contempla principios de salud, ecología y equidad (IFOAM, 2015). 
La agricultura ecológica es un sistema de producción que mantiene y mejora la salud de los suelos, los ecosistemas y las personas. Combina tradición, innovación y la ciencia en beneficio del medio ambiente, promoviendo las relaciones justas y una buena calidad de vida para todos los involucrados (Llano, Morejón \& Pozo, 2014). Por lo que se puede considerar que los productos ecológicos están asociados a múltiples beneficios en la salud de las personas, además son considerados como una alternativa de producción agrícola en armonía con la naturaleza.

Durante las últimas cuatro décadas, la demanda y el consumo de alimentos producidos ecológicamente se ha incrementado de manera significativa, alcanzando los US \$ 38.6 billones en el 2006 (Andrade-Ortiz \& Flores, 2008). En el Ecuador, Los primeros esfuerzos para introducir la agricultura ecológica fueron liderados por ONGs durante la década de los noventa. A partir de entonces, la superficie cultivada se ha incrementado sustancialmente llegando a ocupar el $0.69 \%$ de la superficie total cultivada en el 2011 (IFOAM, 2013). Productos como el cacao, café, banano, caña de azúcar y quinua, los cuales son orientados fundamentalmente a la exportación a países de la Unión Europea y Europa (Andrade-Ortiz \& Flores, 2008).

Sin embargo, desde los países menos desarrollados se han formulado varias críticas al movimiento orgánico y sus preceptos. En primer lugar, desde algunos sectores se cuestiona la legitimidad de la certificación orgánica, conferida principalmente por empresas y organizaciones de los países más desarrollados, como la única forma de asegurar la producción ecológica de alimentos. Se plantea, desde esta perspectiva que muchos agricultores de zonas apartadas de los países desarrollados producen ecológicamente sin recibir ningún precio "premium" como recompensa (Gonzalez \& Nigh, 2005). Además, se fustiga el hecho de que la mayor parte de la producción ecológica se destina a la exportación a países ricos, mientras que la población de los países menos desarrollados continúa consumiendo productos convencionales que se producen con altas dosis de pesticidas.

Ante esto existen varias iniciativas que nacen desde las bases, las cuales asocian a pequeños productores ecológicos quienes comercializan directamente los productos a los consumidores locales, (Chiliguano, 2007). Estas iniciativas son fomentadas por orga- nizaciones gubernamentales y no gubernamentales como una estrategia para por un lado, incrementar los ingresos de los pequeños productores $\mathrm{y}$, por otro, promover el consumo de alimentos libres de pesticidas. Este es el caso de Quito, donde semanalmente tienen lugar varias ferias agroecológicas, las cuales ofrecen al consumidor hortalizas y procesados producidos ecológicamente por agricultores a pequeña escala provenientes de las parroquias rurales colindantes.

Pese a estos antecedentes, son escasos los estudios realizados sobre el consumo de productos ecológicos y sus determinantes entre la población de los países menos desarrollados. El objetivo de este trabajo es analizar cuantitativamente los determinantes socioeconómicos del gasto en productos ecológicos entre los clientes de las ferias agroecológicas del cantón Quito.

El conocer los factores que motivan a un consumidor a gastar más dinero en productos ecológicos reviste gran importancia para diseñadores de políticas que están orientadas a incrementar el consumo de productos ecológicos entre la población ecuatoriana y a la vez, a mejorar los ingresos de pequeños productores.

\section{Materiales y métodos}

\subsection{Muestreo y encuestas}

La principal fuente de datos para el análisis fue una encuesta realizada durante el mes de diciembre de 2014 a consumidores de productos ecológicos, quienes fueron abordados en el parque "La Carolina", en la ciudad de Quito, lugar donde semanalmente tiene lugar una "Feria de productos orgánicos". Se utilizó una técnica de "flujo de poblaciones" (Arthur y Nazroo, 2003), en la cual el encuestador se aproxima a los individuos con las características buscadas en un lugar donde estos tienden a concentrarse. En este caso, se buscaba individuos mayores de edad que consumen productos agroecológicos.

La encuesta utilizada incluyó tres partes, en la primera de las cuales se recogió información demográfica del encuestado y su hogar. La segunda indagó sobre el conocimiento y consumo de productos agroecológicos, hábitos de compra, atributos de los productos orgánicos que consumen, lugar de compra y el tiempo de consumo. 
En la tercera sección se obtuvo información económica del encuestado, sus ingresos y la cantidad asignada mensualmente para alimentación general y el gasto en productos ecológicos. En total se recogieron 101 encuestas.

\subsection{Análisis Estadístico}

Para el análisis estadístico se utilizó una regresión por el método de mínimos cuadrados ordinarios (OLS) de la estructura detallada a continuación:

$$
\mathrm{Y}=\beta_{\mathrm{i}} \mathrm{X}_{\mathrm{i}}+\epsilon_{\mathrm{i}}
$$

Donde $\mathrm{Y}$ es el logaritmo natural del gasto mensual en productos agroecológicos de un individuo i, $\mathrm{X}$ es un vector de variables dependientes, las cuales serán detalladas a continuación, $\beta$ es un vector de coeficientes cuya dirección y magnitud son el objeto de este estudio y $\epsilon$ es el error de la regresión.

El gasto en productos agroecológicos fue modelado como una función de la edad de un individuo, su género, su estado civil, su posición en el hogar, el número de años de educación formal que haya acumulado, su ingreso anual y el número de integrantes del hogar del cual proviene. La Tabla 1 muestra las variables, su definición y sus estadísticas descriptivas.

\section{Resultados}

\subsection{Análisis Descriptivo}

Los datos presentados en la Tabla 1, muestran que los consumidores de productos agroecológicos tienen ingresos mensuales por encima de US \$ 1,500 , cifra que cuadruplica el salario mínimo vital en Ecuador. En promedio, los consumidores de productos agroecológicos exhiben niveles de escolaridad (15.8 años en promedio) considerablemente más altos que los reportados para el resto de la población del Ecuador, lo que indica claramente que quienes consumen productos agroecológicos pertenecen a los segmentos con mayor nivel educativo y con mejor remuneración de la población. En promedio, los integrantes de la muestra gastan US \$ 123 en alimentos agroecológicos.

Tabla 1. Variables, definiciones y estadísticas descriptivas.

\begin{tabular}{llcc}
\hline \multicolumn{1}{c}{ Variable } & \multicolumn{1}{c}{ Definición } & Promedio & Desviación estándar \\
\hline Variable dependiente & & & \\
Gasto & $\begin{array}{l}\text { Logaritmo natural del gasto en } \\
\text { productos agroecológicos }\end{array}$ & 2.995 & 6.214 \\
Variables independientes & Edad del individuo & 46.830 & 15.472 \\
Edad & Edad al cuadrado del individuo & 2,430 & 1,481 \\
Edad al cuadrado & Individuo es mujer & 0.501 \\
Mujer (0/1) & Individuo es soltero & 0.530 & 0.448 \\
Soltero (0/1) & Individuo es jefe de hogar & 0.330 & 3.527 \\
Jefe de hogar (0/1) & Número de años de educación & 15.800 & 11,768 \\
Educación & formal & 18,647 & 1.332 \\
Ingreso anual & Ingreso anual (US \$) & 3.730 & \\
Integrantes & Número de integrantes del hogar & & \\
\hline
\end{tabular}

Nota: $(0 / 1)$ indica que la variables dicotómica

En la Tabla 2, se presenta la relación entre gasto en productos agroecológicos y las motivaciones para adquirirlos. Se observa que casi todos los encuestados, sin importar su nivel de gastos, consumen productos agroecológicos porque consideran que estos son más saludables que los producidos de manera convencional, siendo este patrón más marcado para quienes hacen erogaciones altas (300-500 US \$/mes). Es notorio también que casi ninguno de los encuestados compra alimentos agroecológicos por motivaciones de conservación ambiental. 
Tabla 2. Relación entre gasto en productos agroecológicos (US \$) y motivaciones de compra.

\begin{tabular}{lccc}
\hline & Salud & Estilo de vida & Conciencia ambiental \\
\hline Bajo (0-100 US \$) & $85.1 \%$ & $13.4 \%$ & $1.5 \%$ \\
Medio (100-300 US \$) & $89.3 \%$ & $10.7 \%$ & $0.0 \%$ \\
Alto $(300-500$ US \$) & $100.0 \%$ & $0.0 \%$ & $0.0 \%$ \\
\hline
\end{tabular}

\subsection{Análisis Econométrico}

En la Tabla 3, se presentan los resultados de una regresión por el método de mínimos cuadrados ordinarios. Se aprecia que las variables que influyen sobre el gasto en productos agroecológicos, está definido por las dotaciones de capital financiero (ingresos) y capital humano (escolaridad) de los encuestados. Cada año adicional de educación formal, se incrementa el gasto en alimentos agroecológicos en $5 \%$, mientras que un incremento de US \$ 100 en el ingreso anual de una persona trae como consecuencia un incremento de $1 \%$ en la cantidad gastada en productos ecológicos.

\section{Discusión}

Los resultados aquí presentados indican que, en Quito, el consumo de productos orgánicos está limitado a personas con altos niveles de ingresos y educación formal. Estos resultados están vinculados con estudios previos realizados en otras latitudes, los cuales concluyen que la educación y los ingresos son determinantes claves del consumo de alimentos ecológicos. A diferen- cia de los segmentos menos favorecidos de la población, las personas con mayores ingresos pueden cubrir el "Precio Premium" de los productos agroecológicos (Martinez \& Gazquez, 2007; Salgado-Beltrán, 2011).

Esto es comprensible si se tiene en cuenta que el gasto promedio en productos orgánicos de la muestra (US \$123) equivale al 34\% del salario básico en Ecuador. En el caso de la escolaridad, individuos con mayores dotaciones de educación están más al tanto de los beneficios del consumo de productos libres de pesticidas y de los riesgos que entraña el consumo de alimentos producidos convencionalmente. Es notorio también que la principal motivación para el consumo de productos agroecológicos es que estos son más saludables que los producidos con métodos convencionales (pesticidas y fertilizantes químicos), quedando de lado factores como: conciencia ambiental, equidad, apoyo a los pequeños productores, bienestar animal, entre otros. Este hallazgo está vinculado con el trabajo de López, García \& Barreiro (2008) quienes también reportan que los consumidores ecuatorianos compran alimentos orgánicos porque son más saludables y por sus características organolépticas más que por conciencia ambiental.

Tabla 3. Coeficientes y significancia de la regresión por método de mínimos cuadrados ordinarios (OLS) con el logaritmo natural del gasto en productos agroecológicos como variable dependiente.

\begin{tabular}{|c|c|}
\hline Variable & Coeficiente $(\beta)$ \\
\hline Edad & -0.032 \\
\hline Edad al cuadrado & 0.000 \\
\hline Soltero $(0 / 1)$ & 0.166 \\
\hline Jefe de hogar $(0 / 1)$ & -0.108 \\
\hline Escolaridad & $0.051 * *$ \\
\hline Género $(0 / 1)$ & 0.048 \\
\hline Ingreso anual & $0.001 * * *$ \\
\hline Integrantes & -0.047 \\
\hline Número de observaciones & 101 \\
\hline $\mathrm{R} 2$ & 0.307 \\
\hline Prob $>$ F & 0.000 \\
\hline
\end{tabular}


En términos generales, el consumo de productos agroecológicos es baja, esto se debe a gran medida a que la oferta en el mercado es escasa, razón por la cual los precios tienden a ser considerablemente más altos que los de los productos convencionales. Dado que el consumo de productos ecológicos es impulsado desde organismos gubernamentales (MAGAP, 2013), las políticas públicas deberían orientarse al estímulo y al fomento de los (pequeños) productores agroecológicos a fin de reducir los costos de producción. Esto podría lograrse ofertando asistencia técnica, tecnologías adaptadas al medio y financiamiento con bajos intereses, específicos para productores agroecológicos.

Otro cuello de botella para la masificación del consumo de productos agroecológicos es la escasa difusión sobre los beneficios de su consumo. Más allá de esto, los hallazgos aquí presentados sugieren que casi la totalidad de encuestados consumen alimentos agroecológicos por únicamente por motivos de salud. Ante esto, las campañas publicitarias no deberían enfocarse únicamente en los beneficios individuales (salud) del consumo, sino que también deberían enfatizar en otros aspectos positivos como el apoyo a pequeños productores, protección del medio ambiente, fomento a la equidad, entre otros.

Son escasas las políticas estatales que motiven a la producción y comercialización de productos ecológicos en el Ecuador, la creación de subsidios por parte del gobierno en esta área impulsarían a los agricultores que manejan una producción convencional a incursionar en la producción ecológica para disminuir los costos de los insumos, y del transporte para obtener precios competitivos y al alcance de los consumidores estableciendo así un mercado nacional.

\section{Referencias}

Andrade, D., \& Flores, M. (2008). Consumo de productos organico / agroecologicos en los hogares ecuatorianos.

Arthur, S., \& Nazroo, J. (2003). Designing Fieldwork Strategies and Materials. In J. Ritchie (Ed.), Qualittive
La comercialización de productos ecológicos no se basa simplemente en la compra y venta de estos alimentos sino que encierra a otras variables como la sustentabilidad del medio ambiente, bienestar de los agricultores y la seguridad alimentaria de los consumidores, dándole un sentido más amplio a este segmento de producción, de esta manera se generaría una cultura para el consumo de productos ecológicos.

\section{Conclusiones}

Este estudio ha analizado cuantitativamente los determinantes socioeconómicos del gasto en productos agroecológicos de los compradores de ferias agroecológicas en Quito. Los resultados sugieren que los consumidores de alimentos ecológicos tienen un nivel educativo y un poder adquisitivo considerablemente más alto que la media nacional. El análisis econométrico sugiere que el gasto en alimentos producidos ecológicamente está positivamente correlacionado con el ingreso y el número de años de educación formal, lo que refleja que 1) el consumo de productos ecológicos es restringido para los hogares pobres incapaces de pagar el precio "Premium", y 2) los individuos con mayor educación tienen mayor acceso a información sobre los beneficios que conlleva el consumo de productos ecológicos y los perjuicios que acarrea el consumo de alimentos producidos con pesticidas. Otro hallazgo importante es que casi la totalidad de los encuestados compra productos agroecológicos porque son más saludables que los convencionales, es decir, aspectos como la conciencia ambiental, equidad, apoyo a los pequeños productores, entre otros, no parecen ser incentivos para optar por productos ecológicos.

Research in Practice (pp. 109-137). Thousand Oaks, California: SAGE.

Badii, M., \& Landeros, J. (2007). Plaguicidas que afectan a la salud humana y a la sustentabilidad. 
Chiliguano, L. (2007). Estructuración de un sistema de comercialización de productos orgánicos en el Ecuador.

González, A., \& Nigh, R. (2005) ¿Quién dice qué es orgánico? La certificación y la participación de los pequeños propietarios en el mercado global. Gaceta Ecológica, 77, 19-33.

International Federation of Organic Agriculture Movements-IFOAM (2013). The World of Organic Agriculture. Statistics and Emerging Trends.

International Federation of Organic Agriculture Movements-IFOAM (2015). Principles of Organic Agriculture.

Isan, A. (2013). Diferencias entre los productos biológicos, ecológicos y orgánicos. Obtenido de Ecologismos consumo verde- sostenibilidad garantizada : http://ecologismos.com/diferencias-entre-productos-biologicos-ecologicos-organicos/

Llano, G., Morejón, S. \& Pozo, E. (2014). Análisis del comportamiento del consumidor en las hortalizas orgánicas y diseño estratégico para potenciar el consu- mo en el Distrito Metropolitano de Quito. Obtenido de Universidad Politécnica Salesiana.

Fuentes , C., \& Lopez, E. (2008). El consumo de productos ecológicos. España.

Lopez, B., Garcia, A., \& Barreiro, J. (2013). ¿Conocimiento, medio ambiente o salud? Una investigación sobre los determinantes del consumo de alimentos ecológicos en España. Zaragoza, España.

Martinez, F., Martinez, J., \& Gazquez, L. (2007). Actitudes y comportamientos ambientales: elementos determinantes en el consumo de alimentos ecológicos. Murcia, España.

Ministerio de Agricultura, Ganadería, Acuacultura y Pesca-MAGAP. (2013). Normativa general para promover y regular la producción orgánica-ecológica-biológica en el Ecuador.

Salgado-Beltrán, L. (2011). Factores que influyen en el consumo sustentable de productos orgánicos en el Noroeste de México. Ecosistemas y Productos Agropecuarios, 27(3), 265-279. 